$63^{\text {ème }}$ Congrès de la SFCO, 02011 (2015)

DOI:10.1051/sfco/20156302011

(C) Owned by the authors, published by EDP Sciences, 2015

\title{
COMMUNICATION
}

\section{Utilisation de la membrane amniotique humaine pour régénérer des défauts osseux de calvaria}

\author{
Fenelon $\mathrm{M}^{*}$, Boiziau $\mathrm{C}^{*}$, Tanga $\mathrm{A}^{*}$, Chassande $\mathrm{O}^{*}$, Rey $\mathrm{S}^{*}$, Bœuf $\mathrm{H}^{* *}$, Gindraux $\mathrm{F}^{* * *}$, \\ Ivanovic Z****, Fricain J-C* \\ * INSERM U1026, Université de Bordeaux, Laboratoire BioTis, Bordeaux \\ ** CNRS UMR 5164, Université de Bordeaux, Laboratoire CIRID, Bordeaux \\ *** Université de Franche-Comté, EA 4268, Besançon \\ **** Etablissement Français du Sang Aquitaine-Limousin, Bordeaux
}

Introduction : Le traitement des pertes de substances osseuses segmentaires repose en partie sur l'utilisation de la membrane induite (Swetyenga et al. 2009). Cependant cette membrane présente des limites liées à son absence de pouvoir ostéoinducteur (absence de cellules ostéoprogénitrices et faible quantité de facteurs de croissance (Catros et al. 2009) et à la nécessité d'un deuxième site opératoire (Swetyenga et al. 2012). La membrane amniotique humaine (MAh) pourrait être une alternative à la membrane induite. La MAh contient des cellules stromales mésenchymateuses et des cellules épithéliales (AMSC - AEC) qui ont un fort potentiel ostéogénique in vitro (Soncini 2007). La MAh cryopréservée a été utilisée associée à des substituts osseux pour traiter des furcations dentaires chez l’homme (Kothiwale et al. 2009). Le pouvoir ostéogénique de la MAh a été peu étudié. L’objectif de cette étude est de préciser l'influence des faces épithéliales et mésenchymateuses et de la cryopréservation sur la régénération osseuse de défauts critiques de calvaria de souris.

Matériels et méthodes : In vitro, les MAh fraiches et cryopréservées ont été mises en culture (milieu RPMI, 10 \% SVF) pendant une semaine et des tests, alamarblue et live dead ont été réalisés. In vivo, des défauts de calvaria de 3,3 mm de diamètre ont été réalisés chez des souris OF1 et C57Bl6 adultes. Dix souris par condition ont été utilisées. Les conditions testées étaient : un défaut non comblé et des défauts comblés par de la membrane amniotique fraiche face épithéliale ou face mésenchymateuse au contact du défaut et des défauts comblés par de la membrane amniotique cryopréservée face épithéliale ou face mésenchymateuse au contact du défaut. Les souris ont été sacrifiées 2 mois après le comblement. Les analyses réalisées étaient une radiographie au faxitron, une analyse quantitative au micro scanner $\mathrm{X}$, une analyse histologique.

Résultats : L'activité métabolique des cellules mesurée par le test alamarblue a montré que l'activité métabolique était stable dans la MAh fraiche alors qu'elle diminuait significativement suite à la cryopréservation $(\mathrm{p}<0,05)$. Le test live dead a montré qu'après cryopréservation, lors de la mise en culture, la majorité des cellules épithéliales et mésenchymateuses de la membrane étaient mortes mais qu'après 7 jours de culture, des cellules vivantes en division étaient observées. Après la chirurgie, aucune différence de comportement des animaux entre les différentes conditions n’a été observée. Les résultats de l'analyse au faxitron ont montré qu'aucun défaut n'a été régénéré ad integrum. Des points focaux de minéralisation ont été observés, principalement pour la condition MAh cryopréservée face épithéliale au contact de l'os à réparer. L'analyse quantitative de la minéralisation a confirmé que la différence était significative entre la membrane fraiche ou la membrane cryopréservée et le trou non comblé. L’analyse histologique a confirmé la néoformation osseuse au sein des défauts de calvaria comblés avec la MAh cryopréservée.

This is an Open Access article distributed under the terms of the Creative Commons Attribution License 4.0, which permits unrestricted use, distribution, and reproduction in any medium, provided the original work is properly cited. 
Discussion-conclusion : La cryopréservationaltère la viabilité des cellules de la membrane amniotique. Cependant, les résultats préliminaires indiquent que malgré la cryopréservation, la MAh peut participer à la réparation osseuse.

Nom et adresse du conférencier

Mathilde FENELON

INSERM U1026, Université de Bordeaux

Université de Bordeaux, Laboratoire BioTis

Bordeaux (France)

mathildefenelon@live.fr 